\title{
KÄHLER RICCI FLOW ON FANO MANIFOLDS WITH VANISHED FUTAKI INVARIANTS
}

\author{
ZHENLEI ZHANG
}

\begin{abstract}
In this paper, we establish several sufficient and necessary conditions for the convergence of a Kähler-Ricci flow, on a Kähler manifold with positive first Chern class, to a Kähler-Einstein metric (or a shrinking Kähler-Ricci soliton).
\end{abstract}

\section{Introduction}

Ricci flow has been proved to be an effective method to find Kähler-Einstein metrics on a compact Kähler manifold. In [1], Cao showed the long-time existence of the Kähler-Ricci flow and proved that it will converge to a Kähler-Einstein metric when the first Chern class is nonpositive. For a manifold with positive first Chern class, because of the well-known obstructions to the existence of constant curvature metric, the convergence of the Kähler-Ricci flow becomes complicated and can hopefully be characterized by the Hamilton-Tian's conjecture [11]. However, suppose extra conditions in the later case, many convergence results have been proved by experts. Assuming the existence of Kähler-Einstein metric, Chen and Tian [10,11] proved that the Kähler-Ricci flow will converge to this metric if the bisectional curvature is positive; later, Perelman showed the convergence to this Kähler-Einstein metric without any curvature condition, and this was extended to shrinking Kähler-Ricci solitons by Tian and Zhu [27]. In [15,16,18], Phong et al. studied the Kähler-Ricci flow with two stability conditions-Mabuchi K-energy is bounded from below and the positive eigenvalue of $\bar{\partial}$ operator on $T^{1,0}$ vector fields is uniformly bounded from below-and proved the convergence to Kähler-Einstein metrics; the result was later generalized to shrinking Kähler-Ricci soliton cases by them [17]. When considering the eigenvalue on function space, in $[4,6]$, Chen, $\mathrm{Li}$ and Wang proved the convergence result to Kähler-Einstein metrics under some curvature conditions for the initial metric and a pre-stable condition on the complex structure of the Kähler-Ricci flow. Several stability theorems for Kähler-Ricci flow around a Kähler-Einstein metric (or shrinking Kähler-Ricci soliton) have also been proved independently through different methods by Tian and Zhu [28], Zhu [33], Chen and Li [6], Sun and Wang [22] and Zheng [31]. Besides these, many other results on the convergence of Kähler-Ricci flow on manifolds with positive first Chern class, under different conditions, are obtained; see $[2,3,5,7-9,13,14,19,20,23,29,32]$.

As we have seen, some of the work mentioned above depend more or less on the eigenvalue estimate of Laplace under the Kähler-Ricci flow; see [10, Section 10],

Received by the editors December 24, 2010.

1991 Mathematics Subject Classification. 53C21, 53C25, 53C55.

Key words and phrases. Kähler-Ricci flow, Futaki invariant, eigenvalue. 
[4, Sections 4-6], [6, Sections 3-6], [33, Sections 1-2], [22, Section 3] for details. We remark that the arguments in these works still rely on a strict additional condition, namely the Kähler-Ricci flow should be close to a Kähler-Einstein metric at any time, or its curvature should be uniformly bounded, to derive the convergence of the metric. However, by [15,17], when one considers the convergence of a Kähler-Ricci flow whose eigenvalue of $\bar{\partial}$ operator on vector fields is uniformly bounded away from zero, these extra conditions can be removed if Mabuchi $K$-energy admits a lower bound. Motivated by these observations, in this paper, we want to study how weak the condition on the eigenvalue is possible for the smooth convergence of a Kähelr-Ricci flow. As a result, we find several other sufficient and necessary conditions for the convergence of a Kähler-Ricci flow on manifolds with vanished Futaki invariants; see the theorems in this section and the Remark 5.2 in Section 5.

Now let $\left(M, g_{0}\right)$ be a compact Kähler manifold with $c_{1}(M)>0$. Suppose its Kähler form belongs to $\pi c_{1}(M)$. Let $g(t)$ be the solution to the Kähler-Ricci flow with initial metric $g(0)=g_{0}$ :

$$
\frac{\partial}{\partial t} g_{i \bar{j}}=-R_{i \bar{j}}+g_{i \bar{j}}
$$

By Cao [1], we know that the solution exists for all time $t \in[0, \infty)$. It is obvious that $g(t)$ preserves the Kähler class $\pi c_{1}(M)$ and the volume. Denote by $\mathrm{V}=\int_{M} d v_{g_{0}}$ the volume of this Kähler-Ricci flow. Then, by the $\partial \bar{\partial}$ lemma, there exists a family of smooth real-valued functions $u(t)$ such that

$$
R_{i \bar{j}}(g(t))+\partial_{i} \bar{\partial}_{j} u(t)=g_{i \bar{j}}(t) .
$$

We may suppose that $u(t)$ is normalized such that

$$
\frac{1}{\mathrm{~V}} \int_{M} \mathrm{e}^{-u(t)} d v_{g(t)}=1
$$

where $d v_{g(t)}$ denotes the volume element of the metric $g(t)$. For simplicity, let

$$
a(t)=\frac{1}{\mathrm{~V}} \int_{M} u(t) \mathrm{e}^{-u(t)} d v_{g(t)}
$$

denote the average of $u(t)$ with respect to the probability measure $\frac{1}{\mathrm{~V}} e^{-u(t)} d v_{g(t)}$.

Recall the Poincaré inequality for the Kähler manifold $(M, g(t))$; cf. [12],

$$
\int_{M}|\nabla f|^{2} e^{-u(t)} d v_{g(t)} \geq \int_{M}(f-\bar{f})^{2} e^{-u(t)} d v_{g(t)}, \quad \forall f \in C^{\infty}(M),
$$

where $\bar{f}=\frac{1}{\mathrm{~V}} \int_{M} f e^{-u(t)} d v_{g(t)}$; the equality holds iff $\nabla f=g^{i \bar{j}} \frac{\partial f}{\partial \bar{z}^{j}} \frac{\partial}{\partial z^{i}}$ is a holomorphic vector field. Define an operator $L=-\triangle+g^{i \bar{j}} \nabla_{i} u \nabla_{\bar{j}}$ for each time $t$, then the Poincaré inequality is equivalent to say that the first eigenvalue of $L$ is $\geq 1$.

Our first observation is the following theorem, which states that if the Ricci potential $u(t)$ satisfies a "strict" Poincaré inequality uniformly, then the Kähler-Ricci flow will converge exponentially fast to a Kähler-Einstein metric.

Theorem 1.1. Let $(M, g(t)), t \in[0, \infty)$, be a solution to the Kähler-Ricci flow (1.1) whose Kähler form lies in $\pi c_{1}(M)$ and $u(t)$ be associated Ricci potential satisfying (1.2) and (1.3). If

$$
\text { (1.1) } \int_{M}|\nabla u(t)|^{2} e^{-u(t)} d v_{g(t)} \geq(1+\delta) \int_{M}(u(t)-a(t))^{2} e^{-u(t)} d v_{g(t)}
$$


holds for a uniform constant $\delta>0$ independent of $t$, then $g(t)$ converges exponentially fast in the $C^{\infty}$ sense to a Kähler-Einstein metric.

Remark 1.1. The condition (1.1) has been partially used in [10, Section 10], [4, Section 4] and [33, Section 1-2], together with some curvature conditions under the Kähler-Ricci flow, to derive the exponential decay of $\|u-a\|_{C^{0}}$. We will show in Section 2 that all these extra conditions are actually unnecessary for the same purpose.

Remark 1.2. Although a Poincaré type inequality is needed in the proof, we do not assume the gap estimate on the first eigenvalue of Laplace (or $L$ ). All we need is a weaker condition with regard to the Ricci potential $u$.

Remark 1.3. In Section 5, we will show that the condition (1.1), is actually also necessary for the convergence of the Kähler-Ricci flow to a Kähler-Einstein metric.

Then we can prove the following theorem, which relates the convergence of a Kähler-Ricci flow to the estimate of the "second" eigenvalue of $L$.

Theorem 1.2. Let $(M, g(t)), t \in[0, \infty)$, be a solution to the Kähler-Ricci flow (1.1) whose Kähler form lies in $\pi c_{1}(M)$ and $u(t)$ be associated Ricci potential satisfying (1.2) and (1.3). Let $\lambda(t)$ be the smallest eigenvalue, except 1 , of $L$ acting on smooth functions at time $t$. Suppose that

(2.1) $M$ has vanished Futaki invariant on $\pi c_{1}(M)$, and

(2.2) $\lambda(t) \geq 1+\delta$ for a uniform constant $\delta>0$,

then $g(t)$ converges exponentially fast in the $C^{\infty}$ sense to a Kähler-Einstein metric.

We shall show that the lower bound of the eigenvalue of $\bar{\partial}^{*} \bar{\partial}$ on $T^{1,0}$ vector fields, which was introduced in [18], gives rise to an estimate of $\delta$ in Theorem 1.2. As a consequence, we prove the following generalization of a theorem of Phong et al. [15], where the condition of lower bounded Mabuchi $K$-energy weakened to the vanishing of the Futaki invariant.

Theorem 1.3. Let $(M, g(t)), t \in[0, \infty)$, be a solution to the Kähler-Ricci flow (1.1) whose Kähler form lies in $\pi c_{1}(M)$. Let $\mu(t)$ be the smallest positive eigenvalue of $-g^{i \bar{j}} \nabla_{i} \nabla_{\bar{j}}$ acting on smooth $T^{1,0}$ vector fields at time $t$. Suppose that

(3.1) the Futaki invariant vanishes on $\pi c_{1}(M)$, and

(3.2) $\mu(t) \geq c$ for a uniform constant $c>0$,

then $g(t)$ converges exponentially fast in the $C^{\infty}$ sense to a Kähler-Einstein metric.

Finally, we consider the modified Kähler-Ricci flow. We adopt the notion in [17]. Let $M$ be a compact Kähler manifold with $c_{1}(M)>0$ and $X$ be a holomorphic vector field whose imaginary part $\operatorname{Im}(X)$ induces an $S^{1}$ holomorphic action on $M$. Let $g_{0}$ be an invariant metric whose Kähler class is $\pi c_{1}(M)$. Then the modified Kähler-Ricci flow with respect to the holomorphic vector field $X$, with initial metric $g(0)=g_{0}$, is defined by; cf. [17]:

$$
\frac{\partial}{\partial t} g_{i \bar{j}}=-R_{i \bar{j}}+g_{i \bar{j}}+g_{k \bar{j}} \nabla_{i} X^{k}
$$

This flow preserves the Kähler class as well as the invariance by $S^{1}$ action. Furthermore, the modified flow is just the reparametrization of the Kähler-Ricci flow (1.1) 
by holomorphic transformations generated by $\operatorname{Im}(X)$. Thus, it still exists for all time $t \in[0, \infty)$. By a slight modification, we can prove the similar convergence results for the modified Kähler-Ricci flow; see Section 4 for more details. Here we just state the following generalization of the main theorem of [17], which gives a complete solution to the Question (3) in [17, Section 8].

Theorem 1.4. Let $M$ be a compact Kähler manifold with $c_{1}(M)>0$ and $X$ be a holomorphic vector field. Let $g(t), t \in[0, \infty)$, be a solution to the modified KählerRicci flow with respect to $X$. Let $\mu(t)$ be the smallest positive eigenvalue of $-g^{i \bar{j}} \nabla_{i} \nabla_{\bar{j}}$ acting on smooth $T^{1,0}$ vector fields at time $t$. Suppose that

(4.1) the modified Futaki invariant vanishes on $\pi c_{1}(M)$, and

(4.2) $\mu(t) \geq c$ for a uniform constant $c>0$,

then $g(t)$ converges exponentially fast in the $C^{\infty}$ sense to a Kähler-Ricci soliton with respect to the holomorphic vector field $X$.

We refer to [26] or [17] for the definition and basic properties of the modified Futaki invariant.

The paper is organized as follows: In Section 2, we prove Theorem 1.1; In Section 3, we consider how the Futaki invariant effects the Poincaré inequality and give a proof of Theorem 1.2 and 1.3; In Section 4, we consider the modified Kähler-Ricci flow and prove Theorem 1.4; In Section 5, we give some further remarks.

\section{Kähler-Ricci flow and Poincaré inequality}

Let $\left(M, g_{0}\right)$ be a compact Kähler manifold of dimension $\operatorname{dim}_{\mathbb{C}} M=n$ with associated Kähler form $\omega_{0}$. Suppose $c_{1}(M)>0$ and $\omega_{0} \in \pi c_{1}(M)$. Let $g(t), t \in[0, \infty)$, be the solution to the Kähler-Ricci flow (1.1). Let V be the volume of the Kähler-Ricci flow and $u(t)$ be the normalized Ricci potentials satisfying (1.2) and (1.3). Then it is an easy check that $u(t)$ satisfies the evolution

$$
\frac{\partial}{\partial t} u=\triangle u+u-a,
$$

where $a$ is the average of $u$ defined in (1.4). By Perelman, cf. [21] for a proof, the Ricci potentials $u(t)$ satisfy the uniform bound under the Kähler-Ricci flow:

$$
\|u(t)\|_{C^{0}}+\|\nabla u(t)\|_{C^{0}}+\|\Delta u(t)\|_{C^{0}} \leq C .
$$

Furthermore, the metrics $g(t)$ are uniformly volume noncollapsed:

$$
\operatorname{Vol}_{g(t)}\left(B_{g(t)}(x, r)\right) \geq \kappa r^{2 n}, \quad \forall t>0 \text { and } r \leq 1 .
$$

Here $C$ and $\kappa$ are uniform positive constants depending only on $g_{0}$.

Now we turn to the proof of Theorem 1.1. Several lemmas are needed.

First of all, by a direct computation, cf. Remark (1) in [15, Section 6],

$$
\frac{d a}{d t}(t)=\frac{1}{\mathrm{~V}} \int_{M}\left(|\nabla u|^{2}-(u-a)^{2}\right) e^{-u} d v_{g(t)} .
$$

Introduce

$$
Y=\frac{1}{\mathrm{~V}} \int_{M}(u-a)^{2} e^{-u} d v, \quad Z=\frac{d a}{d t}=\frac{1}{\mathrm{~V}} \int_{M}\left(|\nabla u|^{2}-(u-a)^{2}\right) e^{-u} d v_{g}
$$

at each time $t$. 
Lemma 2.1. For any Kähler-Ricci flow we have $Z(t) \rightarrow 0$ as $t \rightarrow \infty$.

Proof. By Poincaré inequality (1.5), $Z(t) \geq 0$ for any $t$. Then observe that

$$
\int_{0}^{\infty} Z(t) d t=\lim _{t \rightarrow \infty} a(t)-a(0)<\infty .
$$

To show $Z(t) \rightarrow 0$, it suffices to prove that $\frac{d Z}{d t}$ is uniformly bounded from above. Recall that the evolution of $|\nabla u|^{2}$ is given by, cf. [21],

$$
\frac{\partial}{\partial t}|\nabla u|^{2}=\triangle|\nabla u|^{2}-|\nabla \nabla u|^{2}-|\nabla \bar{\nabla} u|^{2}+|\nabla u|^{2}
$$

Thus, by Perelman's estimate (2.2),

$$
\begin{aligned}
\frac{d Z}{d t}= & \frac{d}{d t} \frac{1}{\mathrm{~V}} \int_{M}\left(|\nabla u|^{2}-(u-a)^{2}\right) e^{-u} d v_{g(t)} \\
= & \frac{1}{\mathrm{~V}} \int_{M}\left[\triangle|\nabla u|^{2}-|\nabla \nabla u|^{2}-|\nabla \bar{\nabla} u|^{2}+3|\nabla u|^{2}-\triangle(u-a)^{2}\right. \\
& \left.-2(u-a)^{2}-\left(|\nabla u|^{2}-(u-a)^{2}\right)(u-a)\right] e^{-u} d v \\
= & \frac{1}{\mathrm{~V}} \int_{M}\left[-|\nabla \nabla u|^{2}-|\nabla \bar{\nabla} u|^{2}+3|\nabla u|^{2}-2(u-a)^{2}\right. \\
& \left.+\left(|\nabla u|^{2}-(u-a)^{2}\right)\left(-\triangle u+|\nabla u|^{2}-u+a\right)\right] e^{-u} d v
\end{aligned}
$$

is uniformly bounded from above. Here, in the second equality, we used

$$
\frac{\partial}{\partial t}\left(e^{-u} d v\right)=(-\triangle u-u+a+n-s) e^{-u} d v=-(u-a) e^{-u} d v
$$

since the Ricci potential satisfies $s+\Delta u=n$, where we denote by $s=g^{i \bar{j}} R_{i \bar{j}}$ the complex scalar curvature.

Lemma 2.2. Assume as in Theorem 1.1, then $Y(t) \rightarrow 0$ as $t \rightarrow \infty$.

Proof. Applying the assumption (1.1) to (2.4) gives

$$
Z=\frac{d a}{d t} \geq \frac{\delta}{V} \int_{M}(u-a)^{2} e^{-u} d v
$$

Then use above lemma.

The following lemma gives the $C^{0}$ estimate of $(u-a)$ in terms of its $L^{2}$ estimate through Perelman's gradient estimate.

Lemma 2.3. There exists a constant $A$ depending only on $g_{0}$ such that

$$
\|u-a\|_{C^{0}} \leq A Y^{\frac{1}{2 n+2}}
$$

at any time.

Proof. Combine equation (4.7) in [15] with Perelman's $C^{0}$ estimate of $u(2.2)$.

The following lemma is an essential one in the proof of Theorem 1.1. We remark that it has appeared in $[4,10,11]$ when additional conditions are given. 
Lemma 2.4. Assume as in Theorem 1.1, then there exist positive constants $\gamma$ and $B$ depending on $g(0)$ and $\delta$ such that

$$
Y(t) \leq B e^{-\gamma t}, \quad \forall t \in[0, \infty) .
$$

Proof. By Lemmas 2.2 and 2.3, $\|u-a\|_{C^{0}} \rightarrow 0$ as $t \rightarrow \infty$. Thus,

$$
\begin{aligned}
\frac{d}{d t} Y & =\frac{1}{\mathrm{~V}} \int_{M}\left[2(u-a)\left(\Delta u+u-a-\frac{d a}{d t}\right)-(u-a)^{3}\right] e^{-u} d v \\
& =\frac{1}{\mathrm{~V}} \int_{M}\left[2(u-a)|\nabla u|^{2}-2|\nabla u|^{2}+2(u-a)^{2}-(u-a)^{3}\right] e^{-u} d v \\
& \leq \frac{1}{\mathrm{~V}} \int_{M}\left[\left(-2+2\|u-a\|_{C^{0}}\right)|\nabla u|^{2}+\left(2+\|u-a\|_{C^{0}}\right)(u-a)^{2}\right] e^{-u} d v \\
& \leq\left(\left(-2+2\|u-a\|_{C^{0}}\right)(1+\delta)+\left(2+\|u-a\|_{C^{0}}\right)\right) Y \\
& \leq-\delta \cdot Y,
\end{aligned}
$$

whenever $t$ is large enough, where we used the assumption (1.1) in the second inequality. This suffices to complete the proof of the lemma.

Now we are ready to conclude the proof of Theorem 1.1.

Proof of Theorem 1.1. One can use the argument of [15] to give a proof of the theorem, namely first get the exponential decay of $\triangle u(t)$ by $[15$, Lemma 1] and then apply [15, Lemma 6]. Or, adopt another direct proof as follows.

Let $g(t), t \in[0, \infty)$, be the solution to the Kähler-Ricci flow and $u(t)$ be associated Ricci potentials as above. Define a family of functions

$$
\phi(x, t)=\int_{0}^{t}(u(x, s)-a(s)) d s, \quad \forall x \in M, t \geq 0 .
$$

It is an easy check that $\phi(t)$ is nothing but the relative Kähler potential of $g(t)$ in the sense that $g_{i \bar{j}}(t)=g_{i \bar{j}}(0)+\partial_{i} \partial_{\bar{j}} \phi(t)$. Actually,

$$
\partial_{i} \partial_{\bar{j}} \phi(t)=\int_{0}^{t} \partial_{i} \partial_{\bar{j}} u(s) d s=\int_{0}^{t} \frac{\partial g_{i \bar{j}}}{\partial s} d s=g_{i \bar{j}}(t)-g_{i \bar{j}}(0) .
$$

Combining with Lemmas 2.3 and 2.4 gives the exponential decay of $\|u-a\|_{C^{0}}$. Hence, the potentials $\phi(t)$ is uniformly bounded on $M \times[0, \infty)$. By Yau's resolution of Calabi conjecture [30], see also [24], the metrics $g(t)$ are all $C^{\infty}$ equivalent to each other. By Lemma 1 of $[15],\|\nabla u\|_{C^{0}}$ also decays exponentially, which in turn implies, by the argument in [18] and [15], the exponential decay of $\left\|\frac{\partial g}{\partial t}\right\|_{C^{k}}=\|\operatorname{Ric}-g\|_{C^{k}}$ for any $k$. This completes the proof of the Theorem.

\section{Kähler-Ricci flow and Futaki invariant}

Let $(M, g)$ be any compact Kähler manifold whose Kähler form lies in $\pi c_{1}(M)$ and $u$ be the normalized Ricci potential satisfying (1.2) and (1.3). Denote by $V$ its volume.

Denote by $h^{0}$ the space of holomorphic vector fields on $M$. The Futaki invariant on the Kähler class $\pi c_{1}(M)$, say $\mathrm{F}: h^{0} \rightarrow \mathbb{C}$, is defined via [12,25]

$$
\mathrm{F}(X)=\int_{M} X(u) d v, \quad \forall X \in h^{0} .
$$


It is well known that $\mathrm{F}$ does not depend on the specified chosen metric $g$ in the Kähler class $\pi c_{1}$.

Introduce as in [16] two inner products on the space of sections of $T^{1,0} M$ :

$$
\langle V, W\rangle_{0}=\frac{1}{\mathrm{~V}} \int_{M} g_{i j} V^{i} \overline{W^{j}} d v, \quad\langle V, W\rangle_{u}=\frac{1}{\mathrm{~V}} \int_{M} g_{i \bar{j}} V^{i} \overline{W^{j}} e^{-u} d v .
$$

Denote by $\pi_{0}$ and $\pi_{u}$ the orthogonal projections of $T^{1,0}$ vector fields onto $h^{0}$ with respect to $\langle,\rangle_{0}$ and $\langle,\rangle_{u}$ respectively. Let $\nabla u=g^{i \bar{j}} \frac{\partial u}{\partial \bar{z}^{j}} \frac{\partial}{\partial z^{i}}$ be the complex gradient field of $u$. Then by definition, since $\mathrm{F} \equiv 0$,

$$
\left\langle h^{0}, \nabla u\right\rangle_{0}=0 \text {. }
$$

In particular, as observed by Phong and Sturm in [18],

$$
\int_{M}\left|\pi_{0}(\nabla u)\right|^{2} d v=\mathrm{F}\left(\pi_{0}(\nabla u)\right)=0,
$$

which implies that $\pi_{0}(\nabla u) \equiv 0$. We first establish the following proposition, which relates the second eigenvalue of $L=-\triangle+g^{i \bar{j}} \nabla_{i} u \nabla_{\bar{j}}$ and the strict Poincaré inequality (1.1). The proposition has appeared in $[10,33]$ when the Kähler metric is close to a Kähler-Einstein metric; in [4,6], a similar property was proved for manifolds under certain pre-stable condition.

Proposition 3.1. Let $(M, g)$ be a compact Kähler manifold whose Kähler form lies in $\pi c_{1}(M)$. Suppose the Futaki invariant $\mathrm{F} \equiv 0$ on $\pi c_{1}(M)$. Then the following general estimate holds

$$
\int_{M}|\nabla u|^{2} e^{-u} d v \geq\left(1+\delta^{\prime}\right) \int_{M}(u-a)^{2} e^{-u} d v
$$

where $a=\frac{1}{\mathrm{~V}} \int_{M} u e^{-u} d v$, while $\delta^{\prime}$ is a constant depending only on the lower bound of $\delta$ as in Theorem 1.2 and the upper bound of $\operatorname{osc}(u)$.

Here, $\operatorname{osc}(f)=\max f-\inf f$ for any function $f$. To prove this proposition we need the following lemma.

Lemma 3.1. Let $\nabla u=\pi_{u}(\nabla u)+V$ be the orthogonal decomposition with respect to $\langle,\rangle_{u}$. Then

$$
\left\langle\pi_{u}(\nabla u), \pi_{u}(\nabla u)\right\rangle_{0} \leq\langle V, V\rangle_{0}
$$

Proof. First observe that

$$
0=\left\langle\pi_{u}(\nabla u), \nabla u\right\rangle_{0}=\left\langle\pi_{u}(\nabla u), \pi_{u}(\nabla u)\right\rangle_{0}+\left\langle\pi_{u}(\nabla u), V\right\rangle_{0} .
$$

Then the estimate follows from the Schwarz inequality:

$$
\left\langle\pi_{u}(\nabla u), \pi_{u}(\nabla u)\right\rangle_{0}=-\left\langle\pi_{u}(\nabla u), V\right\rangle_{0} \leq\left\langle\pi_{u}(\nabla u), \pi_{u}(\nabla u)\right\rangle_{0}^{1 / 2} \cdot\langle V, V\rangle_{0}^{1 / 2} .
$$

Now we give a proof of the proposition.

Proof of Proposition 3.1. For brevity define a probability measure $d \rho=\frac{1}{\mathrm{~V}} \mathrm{e}^{-u} d v$ on $M$. Denote by $\lambda_{1}<\lambda_{2}<\cdots$ the sequence of positive eigenvalues of the operator $L=-\triangle+g^{i \bar{j}} \nabla_{i} u \cdot \nabla_{\bar{j}}$ acting on function space $L^{2}(d \rho)$, which are bigger than 1 . In view of the Poincaré inequality (1.5), the only possible eigenvalue of $L$ between 0 and 
$\lambda_{1}$ is $1=\lambda_{0}$ with engenspace generated by functions whose complex gradient fields are holomorphic. Let $E_{k}$ be the eigenspace of $\lambda_{k}$ and

$$
u-a=u_{0}+u_{1}+u_{2}+\cdots
$$

be the unique orthogonal decomposition with respect to the measure $d \rho$, where $u_{k} \in$ $E_{k}$ and $u_{0}$ has holomorphic complex gradient field.

For any $k>0$ we have by assumption $\lambda_{k} \geq \lambda(t) \geq 1+\delta$. Thus,

$$
\begin{aligned}
\int_{M}(u-a)^{2} d \rho & =\sum_{k=0}^{\infty} \int_{M}\left|u_{k}\right|^{2} d \rho=\sum_{k=0}^{\infty} \lambda_{k}^{-1} \int_{M}\left|\nabla u_{k}\right|^{2} d \rho \\
& \leq \int_{M}\left|\nabla u_{0}\right|^{2} d \rho+\sum_{k=1}^{\infty} \frac{1}{1+\delta} \int_{M}\left|\nabla u_{k}\right|^{2} d \rho \\
& =\int_{M}\left(\left|\nabla u_{0}\right|^{2}+\frac{1}{1+\delta}|V|^{2}\right) d \rho .
\end{aligned}
$$

Notice that $\pi_{u}(\nabla u)=\nabla u_{0}$. By Lemma 3.1,

$$
\int_{M}\left|\nabla u_{0}\right|^{2} d \rho \leq \mathrm{e}^{-\min u}\left\langle\nabla u_{0}, \nabla u_{0}\right\rangle_{0} \leq \mathrm{e}^{-\min u}\langle V, V\rangle_{0} \leq \mathrm{e}^{\operatorname{osc}(u)} \int_{M}|V|^{2} d \rho .
$$

Thus, by a simple calculation,

$$
\int_{M}(u-a)^{2} d \rho \leq \int_{M}\left(\left|\nabla u_{0}\right|^{2}+\frac{1}{1+\delta}|V|^{2}\right) d \rho \leq \frac{1+\mathrm{e}^{\mathrm{osc}(u)}+\delta \mathrm{e}^{\mathrm{osc}(u)}}{(1+\delta)\left(1+\mathrm{e}^{\mathrm{osc}(u)}\right)} \int_{M}|\nabla u|^{2} d \rho .
$$

Then one can choose

$$
\delta^{\prime}=\frac{\delta}{1+e^{\operatorname{osc}(u)}+\delta e^{\operatorname{osc}(u)}} .
$$

The proof of the proposition is complete.

With the proposition in hand, then Theorem 1.2 follows directly.

Now we turn to prove Theorem 1.3. For this purpose, we need to reduce the estimate of eigenvalue on vector fields to the estimate of $\lambda$. Denote by $\mu$ and $\tilde{\mu}$ be the lowest positive eigenvalue of $\bar{\partial} * \bar{\partial}$ and $-g^{i \bar{j}} \nabla_{i} \nabla_{\bar{j}}+g^{i \bar{j}} \nabla_{i} u \cdot \nabla_{\bar{j}}$ acting on smooth $T^{1,0}$ vector fields, respectively. Then $\mu$ and $\tilde{\mu}$ can be determined as the largest numbers such that the following hold:

$$
\begin{gathered}
\int_{M}|\nabla V|^{2} d v \geq \mu \int_{M}|V|^{2} d v, \quad \forall\left\langle h^{0}, V\right\rangle_{0}=0 \\
\int_{M}|\nabla V|^{2} e^{-u} d v \geq \tilde{\mu} \int_{M}|V|^{2} e^{-u} d v, \quad \forall\left\langle h^{0}, V\right\rangle_{u}=0,
\end{gathered}
$$

As before we define $\nabla V=g^{i \bar{j}} \nabla_{\bar{j}} V^{k} \frac{\partial}{\partial x^{i}} \otimes \frac{\partial}{\partial z^{k}}$ here. The following lemma is essentially due to Phong et al. [16]:

Lemma 3.2. The eigenvalues $\mu$ and $\tilde{\mu}$ are related by

$$
e^{-\operatorname{osc}(u)} \mu \leq \tilde{\mu} \leq e^{\operatorname{osc}(u)} \mu .
$$


Proof. Let $V$ be a section of $T^{1,0} M$ such that $\left\langle V, h^{0}\right\rangle_{u}=0$ and decompose it with respect to $\langle,\rangle_{0}$ as $V=W+\xi$ such that $\xi \in h^{0}$ and $\langle W, \xi\rangle_{0}=0$. Then,

$$
0=\langle V, \xi\rangle_{u}=\langle\xi, \xi\rangle_{u}+\langle W, \xi\rangle_{u}
$$

Thus, $\langle\xi, \xi\rangle_{u}=-\langle W, \xi\rangle_{u}$. So,

$$
\langle V, V\rangle_{u}=\langle V, W\rangle_{u}=\langle W, W\rangle_{u}+\langle\xi, W\rangle_{u}=\langle W, W\rangle_{u}-\langle\xi, \xi\rangle_{u} \leq\langle W, W\rangle_{u} .
$$

Now, using $\xi \in h^{0}$,

$$
\begin{aligned}
\frac{1}{\mathrm{~V}} \int_{M}|\nabla V|^{2} e^{-u} d v & \geq e^{-\max (u)} \frac{1}{\mathrm{~V}} \int_{M}|\nabla V|^{2} d v=e^{-\max (u)} \frac{1}{\mathrm{~V}} \int_{M}|\nabla W|^{2} d v \\
& \geq \mu e^{-\max (u)}\langle W, W\rangle_{0} \geq \mu e^{-\operatorname{osc}(u)}\langle W, W\rangle_{u} \\
& \geq \mu e^{-\operatorname{osc}(u)}\langle V, V\rangle_{u} .
\end{aligned}
$$

In particular, $\tilde{\mu} \geq \mu \mathrm{e}^{-\operatorname{osc}(u)}$. A similar argument gives another side estimate.

Now we are ready to give a proof of Theorem 1.3.

Proof of Theorem 1.3. It suffices to prove the following estimate for any time:

$$
\lambda \geq 1+e^{-\operatorname{osc}(u)} \mu \text {. }
$$

Actually this follows from an observation in the proof of the Poincaré Lemma. Let $\psi$ be an eigenfunction of $\lambda$, then $\left\langle h^{0}, \nabla \psi\right\rangle_{u}=0$ and thus, cf. Lemma 4.4 in [15],

$$
(\lambda-1) \int_{M}|\nabla \psi|^{2} e^{-u} d v=\int_{M}\left|\nabla_{\bar{i}} \nabla_{\bar{j}} \psi\right|^{2} e^{-u} d v \geq \tilde{\mu} \int_{M}|\nabla \psi|^{2} e^{-u} d v .
$$

Then (3.3) follows directly from Lemma 3.2. The proof of the theorem is complete.

\section{Modified Kähler-Ricci flow by a holomorphic vector field}

Let $M$ be a compact Kähler manifold with $c_{1}(M)>0$ and $X$ be a holomorphic vector field whose imaginary part $\operatorname{Im}(X)$ induces an $S^{1}$ holomorphic action on $M$. Let $g_{0}$ be an invariant metric whose Kähler class is $\pi c_{1}(M)$. Let $g(t), t \in[0, \infty)$, be the solution to the modified Kähler-Ricci flow (1.6) and V be the volume of this flow. Notice that this flow is just a reparametrization of the Kähler-Ricci flow (1.1) starting from $g_{0}$, thus Perelman's estimate of the Ricci potential (2.2) remains valid:

$$
\|u\|_{C^{0}}+\|\nabla u\|_{C^{0}}+\|\triangle u\|_{C^{0}} \leq C \text {. }
$$

Furthermore, the volume noncollapsing condition holds as well:

$$
\operatorname{Vol}_{g(t)}\left(B_{g(t)}(x, r)\right) \geq \kappa r^{2 n}, \quad \forall t>0 \text { and } r \leq 1 .
$$

Here, $C$ and $\kappa$ are positive constant independent of the time.

By Hodge theory, there exists a family of real-valued functions $\theta=\theta_{X}(t)$ such that

$$
\partial_{\bar{j}} \theta=g_{i \bar{j}} X^{i}=X_{\bar{j}} \text {. }
$$

We suppose the functions are normalized by

$$
\frac{1}{V} \int_{M} e^{\theta} d v \equiv 1
$$


Then the modified Kähler-Ricci flow (1.6) can be written as [17]

$$
\frac{\partial}{\partial t} g_{i \bar{j}}=-R_{i \bar{j}}+g_{i \bar{j}}+\partial_{i} \partial_{\bar{j}} \theta \text {. }
$$

As usual, let $u$ be the normalized Ricci potentials determined by (1.2) and (1.3). For simplicity, define as in [17] the modified Ricci potential $w=u+\theta$. Then $g(t)$ is a stationary solution of the modified Kähler-Ricci flow iff $w$ is constant. In [17], the authors also proved similar estimates for $w$ and $\theta$ :

$$
\|\nabla \theta\|_{C^{0}}+\|\triangle \theta\|_{C^{0}}+\|w\|_{C^{0}}+\|\nabla w\|_{C^{0}}+\|\triangle w\|_{C^{0}} \leq C
$$

for some $C$ independent of $t$. In particular, $\|X\|_{C^{0}}$ is uniformly bounded.

Under the normalizing condition (1.3) and (4.4), $\theta$ and $w$ satisfy

$$
\begin{aligned}
\frac{\partial \theta}{\partial t} & =X(w) \\
\frac{\partial w}{\partial t} & =(\triangle+X) w+w-a_{X},
\end{aligned}
$$

where $a_{X}=\frac{1}{\mathrm{~V}} \int_{M} w \mathrm{e}^{-u} d v$ is the average of $w$. Obviously $a_{X}$ is uniformly bounded under the modified Kähler-Ricci flow.

Notice that the modified Kähler-Ricci flow can be written as $\frac{\partial}{\partial t} g_{i \bar{j}}=\partial_{i} \partial_{\bar{j}} w$. Then following the arguments in Section 2 , just by replacing the Ricci potential $u$ by the modified Ricci potential $w$, one can prove the following theorem:

Theorem 4.1. Let $g(t), t \in[0, \infty)$, be a solution to modified Kähler-Ricci flow (1.6) on a compact Kähler manifold $M$ with positive $c_{1}(M)$. Suppose the modified Ricci potential $w(t)$ satisfies

$$
\int_{M}|\nabla w|^{2} e^{-u} d v \geq(1+\delta) \int_{M}\left(w-a_{X}\right)^{2} e^{-u} d v
$$

for some $\delta>0$ independent of $t$, then $g(t)$ converges exponentially fast in the $C^{\infty}$ sense to a Kähler-Ricci soliton.

Here we mention that to show the exponential decay of $\|\nabla w\|_{C^{0}}$ one should use the Smoothing Lemma 10 in [17].

For application, recall the definition of the modified Futaki invariant with respect to the holomorphic vector field $[26], \mathrm{F}_{X}: h^{0} \rightarrow \mathbb{C}$,

$$
\mathrm{F}_{X}(Y)=\int_{M} Y(w) e^{\theta} d v
$$

where $w$ is the modified Ricci potential and $\theta$ is defined via (4.3) and (4.4). It is showed in [26] that the definition does not depend on the specified metric $g$ in $\pi c_{1}(M)$.

Let $\lambda$ be the first eigenvalue, except 1 , of $L=-\triangle+g^{i \bar{j}} \nabla_{i} u \nabla_{\bar{j}}$ acting on function space. As in Section 3, we have the following general proposition:

Proposition 4.1. Let $(M, g)$ be any compact Kähler manifold whose Kähler form lies in $\pi c_{1}(M)$. Suppose the modified Futaki invariant $\mathrm{F}_{X} \equiv 0$ on $\pi c_{1}(M)$. Denote $\lambda=1+\delta$, then the following general estimate holds:

$$
\int_{M}|\nabla w|^{2} e^{-u} d v \geq\left(1+\delta^{\prime}\right) \int_{M}\left(w-a_{X}\right)^{2} e^{-u} d v
$$


where $\delta^{\prime}$ is a constant depending only on the lower bound of $\delta$ and the upper bound of $\operatorname{osc}(w)$.

Proof. Introduce two inner products on the space of sections of $T^{1,0} M$ :

$$
\langle V, W\rangle_{\theta}=\frac{1}{\mathrm{~V}} \int_{M} g_{i \bar{j}} V^{i} \overline{W^{j}} e^{\theta} d v, \quad\langle V, W\rangle_{u}=\frac{1}{\mathrm{~V}} \int_{M} g_{i \bar{j}} V^{i} \overline{W^{j}} e^{-u} d v .
$$

Define a probability measure $d \rho=\frac{1}{\mathrm{~V}} \mathrm{e}^{-u} d v$ and let $w-a_{X}=w_{0}+w_{+}$be an orthogonal decomposition with respect to $d \rho$ where $w_{0}$ has holomorphic complex gradient. In other words, $w_{0}$ is nothing but the projection of $w$ onto the eigenspace of $L$ with eigenvalue 1. Then as in the proof of Proposition 3.1, one can show that

$$
\int_{M}\left(w-a_{X}\right)^{2} d \rho \leq \int_{M}\left(\left|\nabla w_{0}\right|^{2}+\frac{1}{1+\delta}\left|\nabla w_{+}\right|^{2}\right) d \rho .
$$

Next we claim

$$
\int_{M}\left|\nabla w_{0}\right|^{2} d \rho \leq e^{\operatorname{osc}(w)} \int_{M}\left|\nabla w_{+}\right|^{2} d \rho .
$$

Suppose the inequality for the moment, then

$$
\int_{M}\left(w-a_{X}\right)^{2} d \rho \leq \frac{1+e^{\operatorname{osc}(w)}+\delta e^{\operatorname{osc}(w)}}{(1+\delta)\left(1+e^{\operatorname{osc}(w)}\right)} \int_{M}|\nabla w|^{2} d \rho
$$

and thus one can choose $\delta^{\prime}=\frac{\delta}{1+e^{\mathrm{osc}(w)}+\delta e^{\mathrm{osc}(w)}}$.

We finally prove (4.12). Denote by $\pi_{\theta}$ the projection onto $h^{0}$ with respect to $\langle,\rangle_{\theta}$. Since by assumption

$$
\mathrm{F}_{X}\left(\pi_{\theta}(\nabla w)\right)=\left\langle\pi_{\theta}(\nabla w), \nabla w\right\rangle_{\theta}=\left\langle\pi_{\theta}(\nabla w), \pi_{\theta}(\nabla w)\right\rangle_{\theta}=0,
$$

we have $\pi_{\theta}(\nabla w)=0$. Then one can derive as in Lemma 3.2 that

$$
\left\langle\nabla w_{0}, \nabla w_{0}\right\rangle_{\theta} \leq\left\langle\nabla w_{+}, \nabla w_{+}\right\rangle_{\theta} .
$$

Now,

$$
\begin{aligned}
\left\langle\nabla w_{0}, \nabla w_{0}\right\rangle_{u} & \leq e^{-\min w}\left\langle\nabla w_{0}, \nabla w_{0}\right\rangle_{\theta} \leq e^{-\min w}\left\langle\nabla w_{+}, \nabla w_{+}\right\rangle_{\theta} \\
& \leq e^{\operatorname{osc}(w)}\left\langle\nabla w_{+}, \nabla w_{+}\right\rangle_{u}
\end{aligned}
$$

as claimed in (4.12). This completes the proof of the theorem.

As a corollary, one has

Theorem 4.2. Let $(M, g(t)), t \in[0, \infty)$, be a solution to the modified Kähler-Ricci flow (1.6) whose Kähler form lies in $\pi c_{1}(M)$ and $u(t)$ be associated Ricci potential satisfying (1.2) and (1.3). Let $\lambda(t)$ be the smallest eigenvalue, except 1 , of $L$ acting on smooth functions at time $t$. Suppose that

(1) $M$ has vanished modified Futaki invariant on $\pi c_{1}(M)$, and

(2) $\lambda(t) \geq 1+\delta$ for a uniform constant $\delta>0$,

then $g(t)$ converges exponentially fast in the $C^{\infty}$ sense to a Kähler-Ricci soliton with respect to the holomorphic vector field $X$.

Combining with the estimate (3.3), Theorem 1.4 follows directly. 


\section{Further remarks}

Remark 5.1. The average $a$ (respectively $a_{X}$ ) in the Kähler-Ricci flow (respectively modified Kähler-Ricci flow) can be bounded from above by Jensen inequality as follows:

$$
\begin{aligned}
a & =\frac{1}{\mathrm{~V}} \int_{M} u e^{-u} d v \leq \ln \left(\frac{1}{\mathrm{~V}} \int_{M} d v\right)=0 \\
a_{X} & =\frac{1}{\mathrm{~V}} \int_{M} w e^{-u} d v \leq \ln \left(\frac{1}{\mathrm{~V}} \int_{M} e^{w-u} d v\right)=\ln \left(\frac{1}{\mathrm{~V}} \int_{M} e^{\theta} d v\right)=0 .
\end{aligned}
$$

Combining with the monotonicity of $a$ (respectively $a_{X}$ ) under the Kähler-Ricci flow (respectively modified Kähler-Ricci flow), we have the two-sided bound:

$$
a(0) \leq a(t) \leq 0, \quad a_{X}(0) \leq a_{X}(t) \leq 0, \quad \forall t .
$$

Thus, $a$ (respectively $a_{X}$ ) admits a natural bound independent of the $C^{0}$ bound of $u$ (respectively $w$ ) in a prior. The monotonicity of $a$ is proved in [15, Remark (1)]; the monotonicity of $a_{X}$ follows from a similar calculation:

$$
\frac{d a_{X}}{d t}=\frac{1}{\mathrm{~V}} \int_{M}\left(|\nabla w|^{2}-\left(w-a_{X}\right)^{2}\right) e^{-u} d v
$$

which is nonnegative by Poincaré inequality. Furthermore, the increasing of $a_{X}$ is strict unless $g(t)$ is a shrinking Kähler-Ricci soliton.

Remark 5.2. We remark that all the conditions in our theorems on the convergence of the Kähler-Ricci flow are not only sufficient, but also necessary. Indeed, as shown in [15], the convergence of the Kähler-Ricci flow implies the condition (3.1), while the existence of Einstein metric implies the vanishing of the Futaki invariant. The conditions (2.1) and (1.1) follow from condition (3.1) when the Futaki invariant vanishes, as shown in Section 3.

Remark 5.3. The method presented in this paper can also be applied to other problems, such as Questions (1.2) and (1.3) in [6], i.e., the convergence of the KählerRicci flow with an "almost Einstein" initial metric and the stability of the KählerRicci flow around a Kähler-Einstein metric (or more generally a shrinking Kähler-Ricci soliton). Here, "almost Einstein" means that the initial metric is close to an Einstein metric in certain sense. The advantage in our argument is the absence of the pre-stable condition as well as the curvature bounding condition in a prior. We will get back to this point in the future.

\section{Acknowledgments}

The research was initiated when the author was visiting Professor G. Tian in Princeton University in 2009. He author would like to thank the Department of Mathematics for the hospitality; he would like to thank Professor G. Tian for his helpful discussion and constant help. The author also would like to thank Professors D. H. Phong, J. Song, J. Sturm and B. Weinkove for their interests to this paper, especially Professor D. H. Phong for his suggestions to improve the first version of the paper. Thanks also gives K. Zheng for sending his paper [31]. The author thanks Y.G. Zhang for his discussion in the course of writing the paper. Finally the author 
thanks the referee for pointing out the typos and the confusing notation in Section 3. The author is supported by NSF grant 09221010056 of China.

\section{References}

[1] H.-D. Cao, Deformation of Kähler metrics to Kähler-Einstein metrics on compact Kähler manifolds, Invent. Math. 81 (1985), 359-372.

[2] H. D. Cao and M. Zhu, A note on compact Kähler-Ricci flow with positive bisectional curvature, Math. Res. Lett. 16 (2009), 935-939.

[3] X. X. Chen, On the lower bound of energy functional $E_{1}(I)-$ a stability theorem on the Kähler-Ricci flow, J. Geom. Anal. 16 (2006), 23-38.

[4] X. X. Chen, H. Z. Li and B. Wang, Kähler-Ricci flow with small initial energy, Geom. Funct. Anal. 18 (2009), 1525-1563.

[5] X. X. Chen and H. Z. Li, The Kähler-Ricci flow on Kähler manifolds with 2-non-negative traceless bisectional curvature operator, Chin. Ann. Math. 29 (2008), 543-556.

[6] X. X. Chen and H. Z. Li, Stability of Kähler-Ricci flow, J. Geom. Anal. 20 (2010), 306-334.

[7] X. X. Chen and B. Wang, Kähler Ricci flow on Fano surfaces (I), arXiv:0710.5204v2 [math.DG].

[8] X. X. Chen and B. Wang, Remarks on Kähler Ricci flow, J. Geom. Anal. 20 (2010), 335-353.

[9] X. X. Chen and B. Wang, Kähler Ricci flow on Fano manifolds (I), arXiv:0909.2391v2 [math.DG].

[10] X. X. Chen and G. Tian, Ricci flow on Kähler-Einstein surfaces, Invent. Math. 147 (2002), $487-544$.

[11] X. X. Chen and G. Tian, Ricci flow on Kähler-Einstein manifolds, Duke Math. J. 131 (2006), $17-73$.

[12] A. Futaki, Kähler-Einstein metrics and integral invariants, Lecture Notes in Mathematics, 1314 (1988), Springer-Verlag, Berlin.

[13] O. Munteanu and G. Székelyhidi, On convergence of the Kähler-Ricci flow, arXiv:0904.3505v1 [math.DG].

[14] D. H. Phong, N. Sesum and J. Sturm, Multiplier ideal sheaves and the Kähler-Ricci flow, Comm. Anal. Geom. 15 (2007), 613-632.

[15] D. H. Phong, J. Song, J. Sturm and B. Weinkove, The Kähler-Ricci flow and the $\bar{\partial}$ operator on vector fields, J. Diff. Geom. 81 (2009), 631-647.

[16] D. H. Phong, J. Song, J. Sturm and B. Weinkove, The Kähler-Ricci flow with positive bisectional curvature, Invent. Math. 173 (2008), 651-665.

[17] D. H. Phong, J. Song, J. Sturm and B. Weinkove, On the convergence of the modified KählerCRicci flow and solitons, Comment. Math. Helv. 86 (2011), 91-112.

[18] D. H. Phong and J. Sturm, On stability and the convergence of the Kähler-Ricci flow, J. Diff. Geom. 72 (2006), 149-168.

[19] W. D. Ruan, Y. G. Zhang and Z. L. Zhang, Bounding sectional curvature along a Kähler-Ricci flow, Comm. Contem. Math. 11 (2009), 1067-1077.

[20] N. Sesum, Compactness results for the Kähler-Ricci flow, arXiv:0607.2974v4 [math.DG].

[21] N. Sesum and G. Tian, Bounding scalar curvature and diameter along the Kähler Ricci flow (after Perelman), J. Inst. Math. Jussieu 7 (2008), 575-587.

[22] S. Sun and Y. Q. Wang, On the Kähler-Ricci flow near a Kähler-Einstein metric, arXiv: 1004.2018v2 [math.DG].

[23] G. Székelyhidi, The Kähler-Ricci flow and K-polystability, Amer. J. Math. 132 (2010), 1077-1090.

[24] G. Tian, Canonical metrics on Kähler manifolds, Lectures in Mathematics, ETH Zürich, Birkhäuser Verlag, Basel, 2000.

[25] G. Tian, Kähler-Einstein metrics with positive scalar curvature, Invent. Math. 130 (1997), $1-37$.

[26] G. Tian and X. H. Zhu, A new holomorphic invariant and uniqueness of Kähler-Ricci solitons, Comm. Math. Helv. 77 (2002), 297-325.

[27] G. Tian and X. H. Zhu, Convergence of Kähler-Ricci flow, J. Amer. Math. Soc. 20 (2007), 675-699. 
[28] G. Tian and X. H. Zhu, Perelman's W-functional and stability of Kähler-Ricci flow, arXiv:0801.3504v1 [math.DG].

[29] V. Tosatti, Kähler-Ricci flow on stable Fano manifolds, J. Reine Angew. Math. 640 (2010), $67-84$.

[30] S. T. Yau, On the Ricci curvature of a compact Kähler manifold and the complex Monge-Ampè equation, I, Comm. Pure Appl. Math. 31 (1978), 339-411.

[31] K. Zheng, Stability of Kähler-Ricci flow in the space of Kähler metrics, arXiv:1004.2695v2 [math.DG].

[32] X. H. Zhu, Kähler-Ricci flow on a toric manifold with positive first Chern class, arXiv:math/0703486v1 [math.DG].

[33] X. H. Zhu, Stability on Kähler-Ricci flow, I, arXiv:0908.1488v1. [math.DG].

Department of Mathematics, Capital Normal University, Xisanhuan North Road 105, Beijing, 100048, People's Republic of China

E-mail address: zhleigo@yahoo.com.cn 